\title{
The role of patients and healthcare workers Staphylococcus aureus nasal colonization in occurrence of surgical site infection among patients admitted in two centers in Tanzania
}

\author{
Nyambura Moremi ${ }^{1,2^{*}}$ (D) , Heike Claus ${ }^{1}$, Ulrich Vogel ${ }^{1}$ and Stephen E. Mshana ${ }^{2}$
}

\begin{abstract}
Background: Colonization with Staphylococcus aureus has been identified as a risk for subsequent occurrence of infection. This study investigated the relationship between S. aureus colonization of patients and healthcare workers (HCWs), and subsequent surgical site infections (SSI).

Methods: Between December 2014 and September 2015, a total of 930 patients and 143 HCWs were enrolled from the Bugando Medical Centre and Sekou Toure hospital in Mwanza, Tanzania. On admission and discharge nasal swabs, with an additional of wound swab for those who developed SSI were collected from patients whereas HCWs were swabbed once. Identification and antimicrobial susceptibility testing were done by VITEK-MS and VITEK2, respectively. Detection of Panton Valentine leukocidin (PVL) and mecA genes was done by PCR. S. aureus isolates were further characterized by spa typing and Multi-Locus Sequence Typing (MLST).

Results: Among 930 patients screened for S. aureus on admission, 129 (13.9\%) were positive of which 5.4\% (7/129) were methicillin-resistant S. aureus (MRSA). Amongst 363 patients rescreened on discharge, 301 patients had been tested negative on admission of whom 29 (9.6\%) turned positive after their hospital stay. Three (10.3\%) of the 29 acquired S. aureus were MRSA. Inducible Clindamycin resistance occurred more often among acquired S. aureus isolates than among isolates from admission [34.5\% (10/29) vs. 17.1\% (22/129), $P=0.018]$. S. aureus contributed to $21.1 \%(n=12)$ of the 57 cases of investigated SSIs among 536 patients followed. Seven out of eight S. aureus carriage/infection pairs had the same spa and sequence types. The previously reported dominant PVL-positive ST88 MRSA strain with spa type t690 was detected in patients and HCW.

Conclusion: A significant proportion of patients acquired S. aureus during hospitalization. The finding of more than $90 \%$ of S. aureus SSI to be of endogenous source underscores the need of improving infection prevention and control measures including screening and decolonization of high risk patients.
\end{abstract}

Keywords: S. aureus, Colonization, Surgical site infection, Tanzania

\footnotetext{
* Correspondence: nyamburasogone@gmail.com

${ }^{1}$ Institute for Hygiene and Microbiology, University of Wuerzburg, Wuerzburg, Germany

${ }^{2}$ Department of Microbiology and Immunology, Catholic University of Health and Allied Sciences, P. O. Box 1464, Mwanza, Tanzania
}

(c) The Author(s). 2019 Open Access This article is distributed under the terms of the Creative Commons Attribution 4.0 International License (http://creativecommons.org/licenses/by/4.0/), which permits unrestricted use, distribution, and reproduction in any medium, provided you give appropriate credit to the original author(s) and the source, provide a link to the Creative Commons license, and indicate if changes were made. The Creative Commons Public Domain Dedication waiver (http://creativecommons.org/publicdomain/zero/1.0/) applies to the data made available in this article, unless otherwise stated. 


\section{Introduction}

Staphylococcus aureus is a human commensal found most commonly in the anterior nares and in other extranasal sites such as the perineum, skin, pharynx and in small proportion in the gastrointestinal tract and axillae [1-4]. About 20\% of the population is reported to be persistent nasal carriers of S. aureus, 60\% are intermittent carriers and about $20 \%$ are non-carriers [5]. S. aureus has been implicated in a number of clinical infections ranging from minor skin and soft tissue infections to life threatening conditions such as pneumonia and toxic shock syndrome [6].

Colonization of patients with $S$. aureus has been linked to the occurrence of subsequent infections [6]. Under the umbrella of exogenous sources of healthcare-associated infections (HCAIs), healthcare workers (HCWs) have been identified as reservoirs and potential niche for crosstransmission of HCAIs causing pathogens [7]. Colonization and acquisition of methicillin-resistant $S$. aureus (MRSA) during hospital stay not only complicate the management of patients but also significantly affects efforts to prevent and control HCAIs [8].

In Tanzania, surgical site infection (SSI) rates range from 10.9 to $26 \%$ [9-12] and S. aureus is the most isolated pathogen. Despite the predominance of $S$. aureus SSI in Tanzania, the link of patients' colonization and the role of HCWs colonization as possible reservoirs of $S$. aureus have not yet been established. The current study was done to investigate the role of pre-and postadmission $S$. aureus colonization among surgical patients in subsequent occurrence of SSI. In parallel, HCWs attending patients in the same surgical wards were also screened for $S$. aureus nasal colonization to look for evidence of carriers who might act as reservoir and serve as a potential source of $S$. aureus cross transmission.

\section{Methods}

\section{Study design and site}

It was a prospective cohort study conducted for a period of 10 months (December 2014-September 2015) at the Bugando Medical Centre (BMC) and Sekou Toure hospital. Sekou Toure is a regional hospital of Mwanza, it has a bed capacity of 300 and well-established operating theatres. BMC has a bed capacity of 1000 and it serves as a zonal referral hospital caring for about 18 million people residing in 10 out of 30 regions of Tanzania. BMC is also a teaching hospital for the Catholic University of Health and Allied Sciences (CUHAS).

\section{Sample size estimation of the SSI survey}

Kish's formula (1965) was used to estimate the sample size assuming an average prevalence of $18.5 \%$ from the two short clinical studies on SSI done in Mwanza city in
2010 [12] and 2012 [10] whereby a minimum of 230 patients were to be enrolled. However, considering the design effect of the two sites, the estimated sample size was inflated by $10 \%$ which added to 253 as the minimum sample size.

\section{Patients and HCWs}

Consented patients admitted for surgery between December 2014 and September 2015 were enrolled into the study. Patients' social-demographic information and medical history relevant to the study were recorded using a data collection tool. Patients' nasal swabs were taken before surgery within $48 \mathrm{~h}$ of admission to assess S. aureus carriage status before hospital stay. When patients were discharged from the hospital, a second nasal swab was taken to assess $S$. aureus carriage status during hospital stay ( $S$. aureus acquisition). After surgery, patients were followed up by either a surveillance doctor or a trained nurse to observe the signs of infection at the incision site while in the wards. In case of SSI development pus swabs were taken for microbiological investigations [13]. Discharged patients were followedup using their mobile phone numbers until either a SSI became apparent or up to 30 days after being operated. Some of the patients whose surgeries involved foreign body implantation were followed-up for 90 days [13].

In parallel, consented HCWs working in surgical wards were also enrolled. A nasal swab was taken only once to assess their $S$. aureus carriage status.

\section{Sample collection, culture and antimicrobial susceptibility testing}

For nasal swabs, sterile cotton tipped swab (Mast Group Ltd., United Kingdom) was moistened in $0.85 \%$ sterile saline before swabbing, and then introduced into one nostril (nasal vestibule) to a maximum of $1.5 \mathrm{~cm}$ (range swab). The swab was rotated three times around one inner nasal circumference and the same swab was introduced into the second nostril, and the same was repeated as in the first nostril. The swab was inserted into Amies transport medium (Mast Group Ltd., United Kingdom) and transported to the Catholic University of Health and Allied Sciences (CUHAS) laboratory within two hours for processing. For wound infections, the surface of the infected surgical site was first cleaned with normal saline before swabbing [14].

All nasal and wound swabs were inoculated onto Columbia blood agar (OXOID LTD, Basingstoke, Hampshire, England) for $S$. aureus isolation. The plates were incubated aerobically at $37^{\circ} \mathrm{C}$ and examined for growth after $18-24 \mathrm{~h}$. Preliminary S. aureus screening was based on colony morphology i.e. either yellow to golden yellow or creamy-white colonies with or without betahemolysis on blood agar [15]. Colony morphology 
identification was followed by gram-staining, catalase, slide coagulase and DNase tests.

All positive $S$. aureus isolates were further confirmed by VITEK-MS system (bioMérieux, France).

The antimicrobial susceptibility testing was done using automated VITEK-2 system (bioMérieux, France) following manufacturer's instructions. Briefly, a standardized inoculum with $0.5 \mathrm{McF}$ arland-standard was made from pure culture. The test tube with the inoculum was placed into the VITEK-2 cassettes at the Smart Carrier Station $^{\text {ma }}$ to virtually link the sample and the AST-GP632 (VITEK-2 Card specific for S. aureus). Cefoxitin test for MRSA was performed by Kirby disc diffusion method on Mueller-Hinton E- Agar plates (bioMérieux, France). Isolates with zone of inhibition $\leq 21 \mathrm{~mm}$ were phenotypically considered as MRSA and [16] were confirmed by presence of mecA gene.

\section{Amplification of PVL and mecA genes}

Moremi et.al [17], The PVL gene was amplified using lukSF-Forward (5' -ATCATTAGGTAAAATGTCTGGACATGATCCA-3') and lukSF-Reverse (5'-GCATCA AGTGTATTGGATAGCAAAAGC-3'), whereas mecA1Forward (5'GTAGAAATGACTGAACGTCCGATAA-3') and mecA2-Reverse (5'-CCAATTCCACATTGTTTC GGTCTAA-3') primers were used to amplify mecA gene. The known PVL-positive MRSA isolate VA17763 (spa type t008) was used as a positive control. The PCR reaction was carried out in a $50-\mu \mathrm{l}$ volume mixture which included $400 \mathrm{nM}$ of each primer, $1 \mathrm{X}$ buffer II, $200 \mu \mathrm{M}$ of each deoxynucleoside triphosphate, $1.5 \mathrm{mM}$ $\mathrm{MgCl}_{2}$, and $2 \mathrm{U}$ of AmpliTaq Gold DNA polymerase (Thermo Scientific ${ }^{\mathrm{Th}}$, Darmstadt, Germany) and a $4 \mu \mathrm{l}$ $\left(\mathrm{OD}_{600}=0,1\right)$ DNA template.

\section{Spa typing and multilocus sequence typing (MLST)}

Spa typing and MLST was employed to 60 selected $S$. aureus isolates of which 58 were from patients and two were from HCWs. The selection of the $60 \mathrm{~S}$. aureus isolates for typing was based on either they are carriage and infection pairs, isolates from other patients (the same cohort in the same ward) admitted either 2 weeks before or after the occurrence of SSI of patients, as well as all MRSA isolates from patients and HCWs.

Spa typing was performed using the primers 1095Forward (5'-AGACGATCCTTCGGTGA-3') and 1517Reverse (5'-GCTTTTGCAATGTCATTTACTG-3') [18]. The PCR conditions for the primers were; initial denaturation at $95^{\circ} \mathrm{C}$ for $10 \mathrm{~min}, 30$ cycles were run with $94^{\circ}$ for $30 \mathrm{~s}$, annealing at $55^{\circ}$ for $30 \mathrm{~s}$ and extension at $72{ }^{\circ} \mathrm{C}$ for $30 \mathrm{~s}$ each, followed by a final extension step for $10 \mathrm{~min}$ at $72{ }^{\circ} \mathrm{C}$. The PCR products' purification and sequencing were commercially done at Microsynth SeqLab GmbH (Goettingen, Germany). Isolates were assigned to particular spa types using the spa server (http://www.spaserver.ridom.de, Ridom GmbH, Muenster, Germany).

The seven housekeeping genes of the respective bacterial isolates were amplified as per previously described protocol [19]. TraceEdit Pro software (Ridom GmbH, Germany) and SeqMan Pro software (DNASTAR Inc. USA) were used to trim, quality-check and analyze the sequences. Isolates were assigned to their respective sequence types according to MLST website https:// pubmlst.org/saureus.

\section{Data analysis}

Data were entered in Microsoft Excel 2010 and analyzed using STATA-13 software (STATA Corp LP, USA). Categorical variables such as history of admission, history of surgery, history of antibiotic use etc., were presented as proportions and compared using chi-squared test. Continuous variables such as age and duration of hospital stay were presented as medians [inter quartile range (IQR)] and compared using the Wilcoxon-MannWhitney test. To identify factors associated with S. aureus carriage univariate regression followed by backward multivariate logistic regression analysis was done. A Pvalue of less than 0.05 was considered statistically significant.

\section{Ethical approval and consent to participate}

The protocol for this study was reviewed and approved by the Joint Catholic University of Health and Allied Sciences/ Bugando Medical Centre ethics and scientific review committee (CREC/019/2014). All study participants signed an informed written consent.

\section{Results}

Patients' and HCWs S. aureus nasal carriage and antimicrobial susceptibility pattern

Of the 930 patients screened on admission, 129 (13.9\%) were colonized with $S$. aureus of which $5.4 \%$ (7/129) were MRSA. On discharge 363 patients were rescreened of whom 60 (16.5\%) were colonized with $S$. aureus. Among these 29 out of 301 (9.6\%) patients who had been tested negative on admission turned positive during their hospital stay. Three (10.3\%) of the 29 acquired S. aureus were MRSA.

A total of $143 \mathrm{HCW}$ sorking in surgical wards of BMC and Sekou Toure hospital were screened for nasal S. aureus carriage of whom 11 (7.7\%) were positive. Two (1.4\%) of the $143 \mathrm{HCWs}$ were colonized with MRSA.

All S. aureus isolates from patients were susceptible to fusidic acid, linezolid, mupirocin, teicoplanin and vancomycin. Overall, hospital-acquired S. aureus isolates were more resistant to commonly used antimicrobials than isolates from admission with significantly higher rates for erythromycin, rifampicin and tetracycline (Table 1). 
Table 1 Resistance rates of $S$. aureus isolates on admission and discharge

\begin{tabular}{llll}
\hline Antimicrobial agent & $\begin{array}{l}\text { Admission } \\
(N=129) \\
n(\%)\end{array}$ & $\begin{array}{l}\text { Acquired } \\
(N=29) \\
n(\%)\end{array}$ & $P$ value \\
\hline Benzylpenicillin & $124(96.1)$ & $29(100)$ & 0.139 \\
Clindamycin & $2(1.6)$ & $1(3.4)$ & 0.254 \\
Daptomycin & $2(1.6)$ & 0 & 0.750 \\
Erythromycin & $32(24.8)$ & $12(41.4)$ & 0.035 \\
Fusidic acid & 0 & 0 & - \\
Gentamicin & $5(3.9)$ & $1(3.4)$ & 0.548 \\
ICR & $22(17.1)$ & $10(34.5)$ & 0.018 \\
Levofloxacin & $1(0.8)$ & $1(3.4)$ & 0.126 \\
Linezolid & 0 & 0 & - \\
Mupirocin & 0 & 0 & - \\
Rifampicin & $9(6.9)$ & $7(24.1)$ & 0.003 \\
Trimethoprim- & $68(52.7)$ & $19(65.5)$ & 0.105 \\
sulfamethoxazole & & & \\
Tetracycline & $26(20.2)$ & $14(48.3)$ & 0.001 \\
Teicoplanin & 0 & 0 & - \\
Vancomycin & 0 & 0 & - \\
\hline
\end{tabular}

${ }^{a}$ ICR Inducible clindamycin resistance positive

Inducible clindamycin resistance (ICR) occurred more in MRSA isolates than in methicillin-sensitive $S$. aureus (MSSA) isolates $[17.3 \%(31 / 179)$ vs. $60 \%(6 / 10), P=$ 0.001]. ICR also occurred more often among acquired $S$. aureus isolates than in isolates from admission [34.5\% (10/29) vs. $17.1 \%(22 / 129), P=0.018]$.

\section{S. aureus SSI and associated factors}

Of the 536 followed-up patients, 78 (14.6\%) developed SSI. In addition, out of 57 patients with SSI who were available for microbiological investigations, 12 (21.1\%) cases were due to $S$. aureus of which two were infected with MRSA. The other $78.9 \%$ of SSI cases were due to varieties of gram-negative bacteria as detailed in previous publications $[20,21]$. No association was found between S. aureus SSI and colonization status, history of previous hospitalization, use of antibiotics, history of previous surgeries and history of chronic diseases.

\section{Sequence type (ST) distributions, spa types and PVL genes}

MLST was employed to $60 \mathrm{~S}$. aureus (58 from patients i.e. 12 SSI isolates and 46 carriage isolates, and two carriage isolates from $\mathrm{HCWs}$ ). Of the 46 patients' carriage isolates typed, 17 were from admission carriage and 29 were from discharge (13 acquired). Overall ST distribution was diverse in both groups of isolates. Eight STs were detected in the 17 isolates from admission of which ST8 (4/17) and ST5 (4/17) were dominant. Nine STs were detected among 13 acquired isolates typed of which ST152 (3/13) and ST5 (2/13) were predominant (Table 2).

Regarding the $12 \mathrm{~S}$. aureus SSI isolates, eight STs were detected. Identified STs were ST152 (3), ST5 (2), ST612 (2), ST22 (1), ST72 (1), ST88 (1), ST3118 (1) and a new ST4266 (1). The two MRSA isolates from wounds were all typed as ST612. Out of eight S. aureus carriage/infection pair, seven pairs were clonal by bearing the same spa and ST. Three of the seven SSI pairs were due to carriage strains on admission, whereas the four cases of SSI were due to acquired strains (Table 3).

Overall, ST88 was detected in six of all 60 MLST typed isolates of which five were PVL positive including one isolate from BMC HCW with spa type $t 690$.

\section{Discussion}

This study observed the admission and discharge $S$. aureus colonization rate to be 13.9 and $16.5 \%$, respectively. The observed MRSA carriage of $5.4 \%$ on admission falls within a range of 0.2 to $7.2 \%$ observed among hospitalized patients in high-income countries [22-24]. The carriage rates are lower than 21\% reported in Kwazul Natal, South Africa [25]. The difference observed in the two sub-Saharan African countries could be contributed by the nature of patients screened, in South Africa the majority of enrolled patients were HIV positive. HIV infection has been identified as a predictor of $S$. aureus colonization [26].

This study observed acquisition rate of 9.6 and $1 \%$ of $S$. aureus and MRSA, respectively. Although similar data are limited in African setting, the MRSA acquisition in this study is comparable to $1.7 \%$ previously reported in

Table 2 S. aureus sequence type (ST) distribution among admission, hospital acquired and surgical site infection (SSI) isolates

\begin{tabular}{llll}
\hline ST & Admission $(N=17)$ & Acquired $(N=13)$ & SSI $(N=12)$ \\
\hline 8 & 4 & 1 & - \\
5 & 4 & 2 & 2 \\
152 & 3 & 3 & 3 \\
3118 & 2 & 1 & 1 \\
72 & 1 & 2 & 1 \\
88 & 1 & 1 & 1 \\
$4224^{\mathrm{a}}$ & 1 & - & - \\
$4268^{\mathrm{a}}$ & 1 & - & - \\
188 & - & 1 & - \\
508 & - & 1 & - \\
707 & - & 1 & - \\
612 & - & - & 2 \\
22 & - & - & 1 \\
$4266^{*} \mathrm{a}$ & - & - & 1
\end{tabular}


Table 3 Spa and MLST distribution among S. aureus carriage and SSI isolates

\begin{tabular}{|c|c|c|c|c|c|c|c|c|c|c|}
\hline $\mathrm{ID}$ & Hospital & Ward & Region & Type of surgery & $\mathrm{Spa}^{\mathrm{a}}$ & $\mathrm{ST}^{\mathrm{a}}$ & $\mathrm{Spa}^{\mathrm{b}}$ & $\mathrm{ST}^{\mathrm{b}}$ & Spa-SSI & SSI-ST \\
\hline 4 & Bugando & E8 & Mara & ORIF & - & - & - & - & t690 & 88 \\
\hline 26 & Bugando & C6 & Mara & Craniotomy & - & - & t4353 & 72 & t4353 & 72 \\
\hline 67 & Bugando & C6 & Shinyanga & Amputation & - & - & - & - & t355 & 152 \\
\hline 106 & Bugando & C6 & Mwanza & Cholecystectomy & - & - & - & - & t355 & 152 \\
\hline 150 & Bugando & $J 5$ & Mara & CRelease & - & - & t095 & 508 & t1257 & 612 \\
\hline 152 & Bugando & $J 5$ & Shinyanga & ORIF & & & & & t1257 & 612 \\
\hline 191 & Bugando & C9 & Mara & VPShunt & $\mathrm{t} 148$ & 3118 & t223 & 22 & t223 & 22 \\
\hline 379 & Bugando & C6 & Mwanza & Laparatomy & t355 & 152 & $\mathrm{t} 311$ & 5 & $\mathrm{t} 311$ & 5 \\
\hline ST111 & SToure & MAT & Mwanza & Csection & $\mathrm{t} 148$ & 3118 & $\mathrm{t} 148$ & 3118 & t148 & 3118 \\
\hline ST148 & SToure & MAT & Mwanza & Csection & t002 & 5 & t223 & $4266^{*}$ & t223 & $4266^{*}$ \\
\hline ST159 & SToure & MAT & Mwanza & Csection & t002 & 5 & t002 & 5 & t002 & 5 \\
\hline ST302 & SToure & MAT & Mwanza & Csection & t355 & 152 & t355 & 152 & t355 & 152 \\
\hline
\end{tabular}

$a$ admission, $b$ discharge SSI Surgical site infection CRelease contracture release; ORIF; Open Reduction Internal Fixation; Csection Caesarean Section; ST sequence type; *New ST

the United States [27]. Similar to the susceptibility patterns of $S$. aureus isolates reported in several African countries Tanzania inclusive [28], high rates of resistance to penicillin, trimethoprim-sulfamethoxazole and tetracycline were observed among carriage isolates in this study. Overuse and inappropriate prescription of these antimicrobials for various clinical conditions in Tanzania might be the driver for the observed high rates [29].

As observed previously in $S$. aureus isolates from BMC in Mwanza city [30] and those from a National hospital in Dar es Salaam city [31] in Tanzania, rate of ICR was significantly higher in MRSA than MSSA isolates. Similar findings have been reported among admitted patients in Tanzania [32] and Uganda [33]. Furthermore, as previously observed [34], the ICR rate was significantly higher in hospital-acquired than on admission S. aureus isolates. The fact that all SSI-MRSA isolates were hospital-acquired, and that $35 \%$ of acquired isolates were positive for ICR highlights a possibility of failure to treat S. aureus hospital-acquired infections empirically by clindamycin.

This study identified none of the isolates to be resistant to fusidic acid. This finding is within a $0-2 \%$ range of fusidic acid resistance rate observed in most subSaharan African countries [28]. The rare use of this antimicrobial in our setting could explain the observed findings.

The proportion of $S$. aureus causing SSI in the current study $(21.1 \%)$ is within a range of $12-28.6 \%$ reported in different places in Tanzania including BMC where part of the current study took place $[10,12,35]$. The observation of more than $90 \%$ of $S$. aureus SSI in this study were of endogenous source can be explained by likely to occur nasal to wound transmission of $S$. aureus in case of poor hygiene [36].
The $S$. aureus STs $88,5,8$ and 127 that were reported to be predominant in African continent were also detected in this study [37], including ST612 which was previously reported as a rare ST in South Africa [38]. These findings indicate the availability of specific S. aureus clones circulating in Africa.

Of note, the MRSA variant with spa type t7231 and ST1797 which was reported as predominant clone circulating in the same BMC surgical wards in 2010 [17] was not detected in the current study, suggesting either the possibility of outbreak in 2010 or change of S. aureus clones causing wound infections. Most of the ST88 detected were PVL positive as it was observed in BMC and other parts of the African continent [17, 28].

Although the $S$. aureus carriage rate of $7.7 \%$ observed among HCWs is significantly lower compared to $18.3 \%$ reported in Kenya [39], unlike in this study Kenya reported zero MRSA carriage. The difference might be due to the different nature of the study populations with HCWs of various low-risk cadres including physiotherapy in Kenya compared to high-risk nurses in this study who work in surgical wards with frequent exposure to septic/MRSA positive patients.

This study identified one HCW from BMC surgical ward who was colonized with a PVL positive MRSA ST88, previously reported to cause wound infections in the surgical wards of the same hospital [17]. The fact that this nurse has been serving the same surgical ward including when the previous study was carried out points to a possibility of HCWs to be reservoirs of MRSA strains causing infections in patients.

\section{Conclusion}

Despite the large cohort, this study identified a low MRSA carriage among admitted patients and a low 
MRSA SSI. Most of the SSI were due to endogenous MSSA. Although the evidence is low, there is a possibility of HCWs being reservoirs of MRSA strains, a fact which highlights the role of screening them as part of the hospital IPC measures especially during outbreaks.

\section{Abbreviations}

BMC: Bugando Medical Centre; CUHAS: Catholic University of Health and Allied Sciences; HCW: HealthCare Workers; MLST: Multilocus sequence; MRSA: Methicillin-resistant S. aureus; PVL: Panton Valentine leukocidin; SSI: Surgical site infection; ST: Sequence type

\section{Acknowledgements}

The authors thank the technical assistance provided by Vitus Silago and Hezron Basu of CUHAS microbiology laboratory. They are grateful to the key surveillance nurses from BMC hospital (Stella Rujwauka, Grace Ludovick, Maulidi Misanga, Tecla Tumsime and Paul Mvanda) as well as from Sekou Toure hospital (Pili Mbwana Kombo, Flora George Masanja and Anna Paul Lwanji), for their participation in this study.

\section{Authors' contributions}

NM, HC, UV and SEM conceived the idea and designed the study. NM collected data. NM performed preliminary laboratory analysis. NM and HC performed molecular characterization of the isolates. HC, SEM, UV and NM analysed data. NM wrote the manuscript which was reviewed and approved by UV, HC and SEM.

\section{Funding}

This study was supported by funds from the Institute for Hygiene and Microbiology of Wuerzburg, Germany, CUHAS and German Academic Exchange Service (DAAD)

\section{Availability of data and materials}

All data have been included in this manuscript.

\section{Ethics approval and consent to participate}

The Joint CUHAS/BMC research ethics and review committee approved the study protocol with clearance number CREC/019/2014. All patients signed an informed written consent.

\section{Consent for publication}

Not Applicable.

\section{Competing interests}

The authors declare that they have no competing interests.

Received: 11 March 2019 Accepted: 6 June 2019

Published online: 17 June 2019

\section{References}

1. Williams R. Healthy carriage of Staphylococcus aureus: its prevalence and importance. Bacteriol Rev. 1963;27(1):56.

2. Armstrong-Esther C, Smith J. Carriage patterns of Staphylococcus aureus in a healthy non-hospital population of adults and children. Ann Hum Biol. 1976; 3(3):221-7.

3. Ridley M. Perineal carriage of Staph aureus. Br Med J. 1959:1(5117):270

4. Rimland D, Roberson B. Gastrointestinal carriage of methicillin-resistant Staphylococcus aureus. J Clin Microbiol. 1986:24(1):137-8.

5. Parnaby R, O'Dwyer G, Monsey H, Shafi M. Carriage of Staphylococcus aureus in the elderly. J Hosp Infect. 1996;33(3):201-6.

6. Wertheim HF, Melles DC, Vos MC, van Leeuwen W, van Belkum A, Verbrugh HA, Nouwen JL. The role of nasal carriage in Staphylococcus aureus infections. Lancet Infect Dis. 2005:5(12):751-62.

7. Albrich WC, Harbarth S. Health-care workers: source, vector, or victim of MRSA? Lancet Infect Dis. 2008:8(5):289-301.

8. Williams VR, Callery S, Vearncombe M, Simor AE. The role of colonization pressure in nosocomial transmission of methicillin-resistant Staphylococcus aureus. Am J Infect Control. 2009;37(2):106-10.

9. Eriksen $\mathrm{H}$, Chugulu $\mathrm{S}$, Kondo $\mathrm{S}$, Lingaas E. Surgical-site infections at Kilimanjaro christian medical center. J Hosp Infect. 2003;55(1):14-20.
10. Mpogoro FJ, Mshana SE, Mirambo MM, Kidenya BR, Gumodoka B, Imirzalioglu C. Incidence and predictors of surgical site infections following caesarean sections at Bugando medical Centre, Mwanza, Tanzania. Antimicrob Resist Infect Control. 2014;3(1):25.

11. Fehr J, Hatz C, Soka I, Kibatala P, Urassa H, Smith T, Mshinda H, Frei R, Widmer A. Risk factors for surgical site infection in a Tanzanian district hospital: a challenge for the traditional National Nosocomial Infections Surveillance system index. Infect Control Hosp Epidemiol. 2006;27(12): 1401-4.

12. Mawalla B, Mshana SE, Chalya PL, Imirzalioglu C, Mahalu W. Predictors of surgical site infections among patients undergoing major surgery at Bugando medical Centre in Northwestern Tanzania. BMC Surg. 2011;11(1):1.

13. Centers for diseases Cintrol and prevention: National Healthcare Safety Network (NHSN) patient safety component manual; 2018.

14. Miller JM, Binnicker MJ, Campbell S, Carroll KC, Chapin KC, Gilligan PH, Gonzalez MD, Jerris RC, Kehl SC, Patel R. A guide to utilization of the microbiology laboratory for diagnosis of infectious diseases: 2018 update by the Infectious Diseases Society of America and the American Society for Microbiology. Clin Infect Dis. 2018:67(6):e1-e94.

15. Winn WC, Koneman EW. Koneman's color atlas and textbook of diagnostic microbiology: Lippincott Williams \& wilkins; 2006.

16. Wayne P. CLSI document M02-A11 and M07-A9. Clinical and Laboratory Standards Institute; 2012

17. Moremi N, Mshana SE, Kamugisha E, Kataraihya J, Tappe D, Vogel U, Lyamuya EF, Claus H. Predominance of methicillin resistant Staphylococcus aureus-ST88 and new ST1797 causing wound infection and abscesses. J Infect Dev Ctries. 2012;6(08):620-5.

18. Harmsen D, Claus H, Witte W, Rothgänger J, Claus H, Turnwald D, Vogel U. Typing of methicillin-resistant Staphylococcus aureus in a university hospital setting by using novel software for spa repeat determination and database management. J Clin Microbiol. 2003:41(12):5442-8.

19. Enright MC, Day NP, Davies CE, Peacock SJ, Spratt BG. Multilocus sequence typing for characterization of methicillin-resistant and methicillin-susceptible clones of Staphylococcus aureus. J Clin Microbiol. 2000;38(3):1008-15.

20. Moremi N, Claus H, Rutta L, Frosch M, Vogel U, Mshana S. High carriage rate of extended-spectrum beta-lactamase-producing Enterobacteriaceae among patients admitted for surgery in Tanzanian hospitals with a low rate of endogenous surgical site infections. J Hosp Infect. 2018;100(1):47-53.

21. Moremi N, Claus H, Vogel U, Mshana SE. Surveillance of surgical site infections by Pseudomonas aeruginosa and strain characterization in Tanzanian hospitals does not provide proof for a role of hospital water plumbing systems in transmission. Antimicrob Resist Infect Control. 2017; 6(1):56.

22. Barakate M, Yang Y, Foo S, Vickery A, Sharp C, Fowler L, Harris J, West R, Macleod C, Benn R. An epidemiological survey of methicillin-resistant Staphylococcus aureus in a tertiary referral hospital. J Hosp Infect. 2000;44(1): 19-26.

23. Jernigan JA, Clemence MA, Stott GA, Titus MG, Alexander $\mathrm{CH}$, Palumbo CM, Farr BM. Control of methicillin-resistant Staphylococcus aureus at a university hospital: one decade later. Infect Control Hosp Epidemiol. 1995:16(12):686-96

24. Cohen SH, Morita MM, Bradford M. A seven-year experience with methicillin-resistant Staphylococcus aureus. Am J Med. 1991:91(3):S233-7.

25. Heysell SK, Shenoi SV, Catterick K, Thomas TA, Friedland G. Prevalence of methicillin-resistant Staphylococcus aureus nasal carriage among hospitalised patients with tuberculosis in rural Kwazulu-Natal. S Afr Med J. 2011;101(5): 332-4.

26. Hidron Al, Kourbatova EV, Halvosa JS, Terrell BJ, McDougal LK, Tenover FC, Blumberg HM, King MD. Risk factors for colonization with methicillinresistant Staphylococcus aureus (MRSA) in patients admitted to an urban hospital: emergence of community-associated MRSA nasal carriage. Clin Infect Dis. 2005:41(2):159-66.

27. Fishbain JT, Lee JC, Nguyen HD, Mikita JA, Mikita CP, Uyehara CF, Hospenthal DR. Nosocomial transmission of methicillin-resistant Staphylococcus aureus: a blinded study to establish baseline acquisition rates. Infect Control Hosp Epidemiol. 2003;24(6):415-21.

28. Schaumburg F, Alabi A, Peters G, Becker K. New epidemiology of Staphylococcus aureus infection in Africa. Clin Microbiol Infect. 2014;20(7): 589-96.

29. Gwimile JJ, Shekalaghe SA, Kapanda GN, Kisanga ER. Antibiotic prescribing practice in management of cough and/or diarrhoea in Moshi municipality, 
northern Tanzania: cross-sectional descriptive study. Pan African Med J. 2012;12(1). https://www.ncbi.nlm.nih.gov/pubmed/23133703.

30. Mshana S, Kamugisha E, Miramb M, Chalya P, Rambau P, Mahalu W, Lyamuya E. Prevalence of clindamycin inducible resistance among methicillin-resistant Staphylococcus aureus at Bugando medical Centre, Mwanza, Tanzania. Tanzan J Health Res. 2009;11(2). https://www.ajol.info/ index.php/thrb/article/view/45197.

31. Moyo SJ, Aboud S, Blomberg B, Mkopi N, Kasubi M, Manji K, Lyamuya EF, Maselle SY, Langeland N. High nasal carriage of methicillin-resistant Staphylococcus aureus among healthy Tanzanian under-5 children. Microb Drug Resist. 2014;20(1):82-8.

32. Moyo SJ, Nkinda L, Majigo M, Mbaga E, Mbembati N, Aboud S, Lyamuya EF. Prevalence of methicillin-resistant Staphylococcus aureus carriage on admission among patients attending regional hospitals in Dar Es Salaam, Tanzania. BMC Res Notes. 2017;10(1):417.

33. Seni J, Bwanga F, Najjuka CF, Makobore P, Okee M, Mshana SE, Kidenya BR, Joloba ML, Kateete DP. Molecular characterization of Staphylococcus aureus from patients with surgical site infections at Mulago Hospital in Kampala, Uganda. PLoS One. 2013;8(6):e66153.

34. Patel M, Waites KB, Moser SA, Cloud GA, Hoesley CJ. Prevalence of inducible clindamycin resistance among community-and hospital-associated Staphylococcus aureus isolates. J Clin Microbiol. 2006;44(7):2481-4.

35. Manyahi J, Matee MI, Majigo M, Moyo S, Mshana SE, Lyamuya EF. Predominance of multi-drug resistant bacterial pathogens causing surgical site infections in Muhimbili national hospital, Tanzania. BMC Res Notes. 2014;7(1):500.

36. Altemeier WA, Culbertson WR, Hummel RP. Surgical considerations of endogenous infections-sources, types, and methods of control. Surg Clin N Am. 1968;48(1):227-40.

37. Breurec $S$, Zriouil $S$, Fall C, Boisier P, Brisse S, Djibo S, Etienne J, Fonkoua M, Perrier-Gros-Claude J, Pouillot R. Epidemiology of methicillin-resistant Staphylococcus aureus lineages in five major African towns: emergence and spread of atypical clones. Clin Microbiol Infect. 2011;17(2):160-5.

38. Jansen van Rensburg M, Eliya Madikane V, Whitelaw A, Chachage M, Haffejee S, Gay Elisha B. The dominant methicillin-resistant Staphylococcus aureus clone from hospitals in Cape Town has an unusual genotype: ST612. Clin Microbiol Infect. 2011;17(5):785-92.

39. Omuse G, Kariuki S, Revathi G. Unexpected absence of meticillin-resistant Staphylococcus aureus nasal carriage by healthcare workers in a tertiary hospital in Kenya. J Hosp Infect. 2012;80(1):71-3.

\section{Publisher's Note}

Springer Nature remains neutral with regard to jurisdictional claims in published maps and institutional affiliations.

Ready to submit your research? Choose BMC and benefit from:

- fast, convenient online submission

- thorough peer review by experienced researchers in your field

- rapid publication on acceptance

- support for research data, including large and complex data types

- gold Open Access which fosters wider collaboration and increased citations

- maximum visibility for your research: over $100 \mathrm{M}$ website views per year

At BMC, research is always in progress.

Learn more biomedcentral.com/submissions 